\title{
Depth-Time Interpolation of Feature Trends Extracted from Mobile Microelectrode Data with Kernel Functions
}

\author{
Stephen Wong ${ }^{\text {a }}$ Eric L. Hargreaves ${ }^{b}$ Gordon H. Baltuch ${ }^{c}$ Jurg L. Jaggi ${ }^{c}$ \\ Shabbar F. Danish ${ }^{b}$ \\ a Department of Neurology, and b ${ }^{b}$ Division of Neurosurgery, Department of Surgery, UMDNJ - Robert Wood

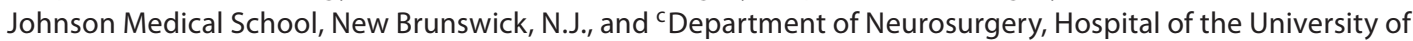 \\ Pennsylvania, Philadelphia, Pa., USA
}

\section{Key Words}

Deep brain stimulation - Microelectrode recording · Intraoperative neurophysiology · Signal processing • Parkinson's disease $\cdot$ Subthalamic nucleus $\cdot$ Kernel functions

\begin{abstract}
Background/Aims: Microelectrode recording (MER) is necessary for precision localization of target structures such as the subthalamic nucleus during deep brain stimulation (DBS) surgery. Attempts to automate this process have produced quantitative temporal trends (feature activity vs. time) extracted from mobile MER data. Our goal was to evaluate computational methods of generating spatial profiles (feature activity vs. depth) from temporal trends that would decouple automated MER localization from the clinical procedure and enhance functional localization in DBS surgery. Methods: We evaluated two methods of interpolation (standard vs. kernel) that generated spatial profiles from temporal trends. We compared interpolated spatial profiles to true spatial profiles that were calculated with depth windows, using correlation coefficient analysis. Results: Excellent approximation of true spatial profiles is achieved by interpola-
\end{abstract}

tion. Kernel-interpolated spatial profiles produced superior correlation coefficient values at optimal kernel widths $(r=$ 0.932-0.940) compared to standard interpolation ( $r=0.891)$. The choice of kernel function and kernel width resulted in trade-offs in smoothing and resolution. Conclusions: Interpolation of feature activity to create spatial profiles from temporal trends is accurate and can standardize and facilitate MER functional localization of subcortical structures. The methods are computationally efficient, enhancing localization without imposing additional constraints on the MER clinical procedure during DBS surgery.

Copyright $\odot 2012$ S. Karger AG, Basel

\section{Background}

Clinical neurophysiological tests involve localization of interesting events from neurophysiological data, such as identification of spikes and seizures from an electroencephalogram $[1,2]$. In these cases, electrodes are typically stationary with respect to signal sources. In contrast, during microelectrode recording (MER) for deep brain stimulation (DBS) surgery, an electrode is moved through various brain structures to localize subcortical

\section{KARGER}

Fax +4161306 1234 E-Mail karger@karger.ch www.karger.com
(C) 2012 S. Karger AG, Base

1011-6125/12/0901-0051\$38.00/0

Accessible online at:

www.karger.com/sfn
Stephen Wong, MD

Department of Neurology

UMDNJ - Robert Wood Johnson Medical School

125 Paterson Street, 6th floor, New Brunswick, NJ 08901 (USA)

Tel.+1 732235 6433,E-Mail wongst@umdnj.edu 
targets for electrical stimulation [3, 4]. This presents a unique situation where signals are acquired in time, yet localization occurs in space.

Attempts to automate the localization process typically involve extracting quantitative features from the microelectrode data and plotting feature activity versus depth (referred to here as a spatial profile). Investigators create these spatial profiles by pausing the microelectrode at regular depth intervals, waiting for the recording to stabilize before extracting features, and plotting these features according to the electrode position [5-9]. Furthermore, many state-of-the art quantitative features require spike sorting to isolate single units and extract their firing characteristics, which are then matched to a lexicon of known patterns [8]. The process of spike sorting also requires a stationary electrode, and having to pause the electrode at intervals to extract features (1) adds time and complexity to the DBS operation and (2) interferes with the clinical task of microelectrode localization.

Recent work indicates that both spike-dependent and spike-independent features contain information that is helpful for subcortical target localization [7, 10-13]. These features are extracted from mobile microelectrodes, bypassing the problem of spike sorting and allowing neurophysiologists to move the microelectrode as they see fit. Though this optimizes the traditional clinical procedure of microelectrode-based target localization, the resultant neurophysiological data are irregularly sampled in space because of (1) electrode slowing near critical areas and (2) electrode pauses during clinical testing. The automated feature output (typically extracted via temporal data windows in a sliding window paradigm) is similarly irregularly sampled, such that one must examine a temporal trend (time vs. feature activity), as well as a separate timeversus-depth plot [14], to localize a target. This adds complexity to the localization task.

Though target localization is easier with spatial profiles, there are advantages of temporal trends. For example, it is reassuring to see a change in feature activity during sensorimotor testing while the electrode is stationary [3]. These changes may not be resolvable on a spatial profile, as averaged signals from a common depth can wash out any signals obtained during sensorimotor testing. It is ideal to have a singular, flexible method to transition between both temporal and spatial profiles to gain the advantages of both structural and functional 'imaging'.

While true spatial profiles can be calculated with sliding depth windows, interpolated spatial profiles can be quickly calculated from temporal trends. The aims of this study are (1) to show quantitatively that reliable spatial profiles can be interpolated from temporal trends and (2) to compare different methods of interpolation to accomplish this. Of the interpolation methods we studied, kernel methods accomplished this in an especially elegant manner and resulted in the closest match between true and interpolated spatial profiles.

\section{Methods}

Clinical Procedure and Data Collection

The MER data used here (44 implanted tracks from 27 patients) were selected from an IRB-approved, de-identified database of digital recordings (24-kHz sampling frequency with 16-bit vertical resolution, hardware filter settings $0.1-10,000 \mathrm{~Hz}$ ). Details of our clinical procedure and data acquisition are described elsewhere [15]. In most cases, the microelectrode trajectory penetrated the following structures: anterior thalamus, zona incerta, $\mathrm{H} 2$ field of Forel, subthalamic nucleus (STN), and substantia nigra (SN). A trained clinical neurophysiologist marked all anatomic boundaries intraoperatively.

Feature Extraction from Sliding Time and Depth Windows

All computational analyses were performed in Matlab (Mathworks, Inc., Natick, Mass., USA). Digitized MER signals were preprocessed by band-pass filtering between 0.5 and $8 \mathrm{kHz}$ with a 6-pole Butterworth filter. Data were divided up into either (1) $50 \%$ overlapping time windows of duration $\mathbf{T}$ or (2) $50 \%$ overlapping depth windows of length $\mathbf{D}$ for feature extraction. We used a 4-second duration for $\mathbf{T}$, and the following lengths for $\mathbf{D}: 0.025$, $0.05,0.075,0.1,0.15$, and $0.2 \mathrm{~mm}$. Our value for $\mathbf{T}$ was based on observations that indicated that this duration provides an empirically optimal trade-off between increased spatial resolution and feature stability at the electrode velocities we typically employed ([14], data not shown). For each window, we used the time and depth at the temporal center of the window as the time and depth stamp.

The computational features used here were reported previously $[14,16]$. We made a slight modification by normalizing each feature to the data window length (e.g. a spike count feature became spike rate). This allowed us to directly compare feature trends calculated via temporal and spatial methods. Additionally, we made another modification to our features that are dependent upon spike detection. Our spike detector previously detected spikes from an adaptive threshold that was based upon the standard deviation of a block of data within a fixed temporal window; we changed the adaptive threshold here to a continuous running standard deviation.

\section{Nonkernel or Standard Interpolation}

We performed linear, cubic spline, and cubic interpolation of temporal trends with Matlab's built-in 1-D interpolation function after averaging points from common depths.

Kernel Interpolation

Kernel functions are even, real-valued probability density functions with unit areas under the curve that are used to perform 
local weighted averaging. The formulas for the kernels used here are shown below and plotted in figure 1:

1. Epanechnikov

$$
K(x, \mu, \lambda)=\left\{\begin{array}{c}
\frac{3}{4}\left(1-\left(\frac{x-\mu}{\lambda}\right)^{2}\right), \frac{|x-\mu|}{\lambda} \leq 1 \\
0, \frac{|x-\mu|}{\lambda}>1
\end{array}\right.
$$

where: $\mu$ is the center of the kernel function; $x$ is the point where we wish to compute the kernel function value, and $\lambda$ is the kernel width.

\section{Gaussian}

$$
K(x, \mu, \lambda)=\frac{1}{2 \pi} e^{-\frac{1}{2}\left(\frac{x-\mu}{\lambda}\right)^{2}}
$$

3. Tricube

$$
K(x, \mu, \lambda)=\left\{\begin{array}{c}
\frac{70}{81}\left(1-\left(\frac{x-\mu}{\lambda}\right)^{3}\right)^{3}, \frac{|x-\mu|}{\lambda} \leq 1 \\
0, \frac{|x-\mu|}{\lambda}>1
\end{array}\right.
$$

4. Uniform

$$
K(x, \mu, \lambda)=\left\{\begin{array}{l}
\frac{1}{2 \lambda}, \frac{|x-\mu|}{\lambda} \leq 1 \\
0, \frac{|x-\mu|}{\lambda}>1
\end{array}\right.
$$

Note that the standard deviation is the kernel width for the Gaussian kernel, while the kernel width represents the local neighborhood half-lengths for the remaining kernel functions.

To create kernel-interpolated spatial profiles, we used the following standard procedure [17]:

1. We have $X=\left\{x_{1}, x_{2}, \ldots, x_{i}, \ldots, x_{n}\right\}$, an n-element vector of irregularly spaced depth values, and $Y=\left\{y_{1}, y_{2}, \ldots, y_{i}, \ldots, y_{n}\right\}$, the corresponding n-element vector of feature values or automated classifier outputs, and $\hat{X}=\left\{\hat{x}_{1}, \hat{x}_{2}, \ldots, \hat{x}_{i}, \ldots, \hat{x}_{n}\right\}$, an m-element vector of regularly spaced depth values for which we wish to interpolate $\hat{Y}$. Select a kernel function $\mathbf{K}$ and a kernel width $\lambda$ that represents the size of the local neighborhood for local-weighted averaging.

2 . For each $\hat{x}_{i}$ in $\hat{X}$, calculate $w_{i}=\left\{w_{i 1}, w_{i 2}, \ldots, w_{i j}, \ldots, w_{i n}\right\}$, the $\mathrm{n}$-element row vector of kernel weights by following:

$$
w_{i j}=K\left(x_{j}, \hat{x}_{i}, \lambda\right)
$$

For each $\hat{x}_{i}$, in $\hat{X}$, calculate the kernel-weighted average value $\hat{y}_{i}$ by following:

$$
\hat{y}_{i}=\frac{W_{i} Y}{\sum W_{i}}
$$

\section{Feature Profile Statistical Comparison}

We compared true versus interpolated spatial profiles with the Pearson correlation coefficient $(r)$ as our quantitative measure. The values for $r$ can vary from -1 (perfect anticorrelation) to 0 (no correlation) to 1 (perfect correlation).

Depth Interpolation of Microelectrode Feature Trends

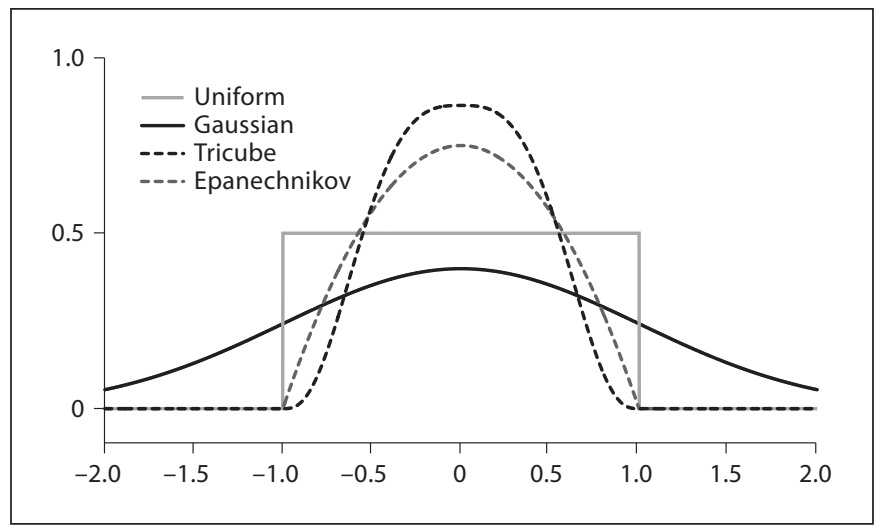

Fig. 1. Typical kernel functions. Four typical kernel functions used in the manuscript to perform local-weighted averaging are shown here, standardized to a kernel width of $\lambda=1$. Each function is even and real-valued with an area under the curve equal to 1. Note that values outside of the kernel width for the Tricube, Epanechnikov, and Uniform kernel functions are zero, while the Gaussian has infinite representation. The Uniform kernel is the only noncontinuous function. The Tricube and Gaussian kernels are differentiable at all points, while the others are not differentiable at the kernel width boundary.

\section{Results}

Summary statistics of our database of 44 tracks are as follows: the mean track length was $15.29 \pm 1.95 \mathrm{~mm}$. The mean record duration was $20.20 \pm 4.40 \mathrm{~mm}$. The mean velocity of our microelectrode was $0.0125 \mathrm{~mm} / \mathrm{s}$.

Figure 2 shows the results of various methods of interpolation on a typical temporal trend (created using 50\% overlapping, 4-second time windows). A true spatial profile (created using 50\% overlapping, $0.075 \mathrm{~mm}$ depth windows) is shown for comparison. The process of localization with a temporal trend requires a separate timeversus-depth plot, shown in figure $2 \mathrm{a}$. Subsequent panels show several interpolated spatial profiles generated via various methods. In general, excellent visual agreement can be achieved between true and interpolated spatial profiles, thereby validating the interpolation approach. Among the kernel interpolation (KI) methods, different kernels give largely similar results; however, because the Gaussian kernel is nonzero outside of $\lambda$, its smoothing effect is more pronounced for the same $\lambda$ (fig. 2b). Figure $2 c$ shows the results of varying $\lambda$; as expected, greater kernel widths result in increased smoothing.

A zoom of the STN transition zone is shown in figure $2 \mathrm{~d}$. Standard interpolation (SI) methods with linear, cubic spline, and cubic methods consider only two adjacent 


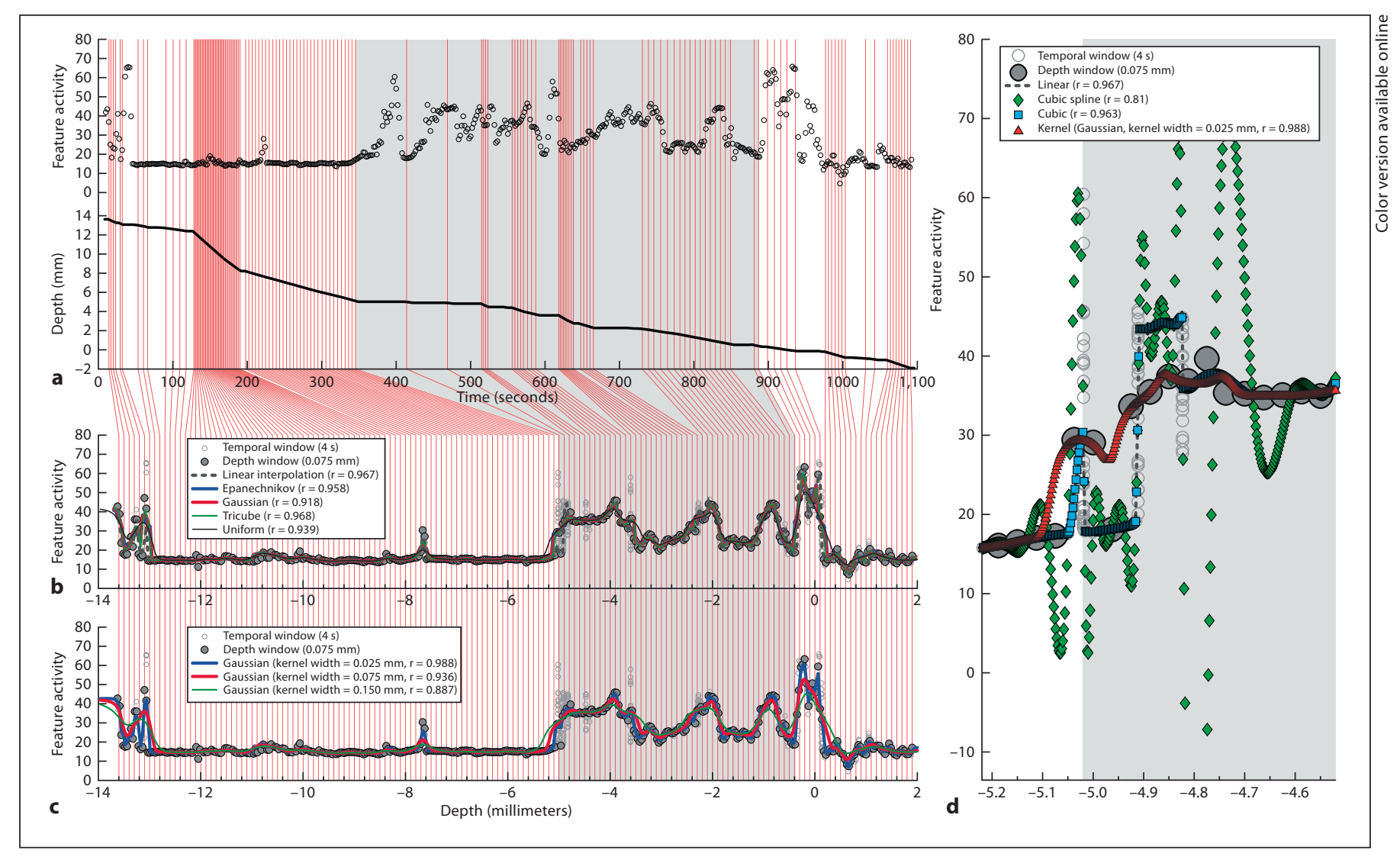

Fig. 2. Spatial profiles generated by interpolation. a shows feature activity (curve length in this case), extracted from raw microelectrode data with 50\% overlapping, 4 -second windows, and plotted against time. A separate time vs. depth trace is required for localization. The gray regions (see color version online) represent the subthalamic nucleus, the operative target in this case. b-d show various spatial profiles. Open light gray circles show direct plotting of feature activity vs. their depthstamps. Filled gray circles represent true spatial profiles generated with $50 \%$ overlapping, $0.075 \mathrm{~mm}$ spatial windows. In $\mathbf{b}$, various solid lines represent kernel-interpo-

points regardless of their depth separation. KI, on the other hand, is 'spatially aware', and considers feature points within a depth neighborhood, thereby better mimicking depth windows and resulting in a generally improved match.

\section{Determining the Optimal D}

To compare true and interpolated spatial profiles, we first determined the appropriate depth window $\mathrm{D}$ for the comparison. Since the average distance travelled per 4 -second time window was $0.0501 \mathrm{~mm}$, the initial assumption is that $\mathbf{D}=0.05 \mathrm{~mm}$ would have resulted in the closest match. We varied $\mathbf{D}$ among the following values: $0.025,0.05,0.075,0.1,0.15$, and $0.2 \mathrm{~mm}$. Correlation coef- lated spatial profiles, created with various kernel functions with kernel width of $0.1 \mathrm{~mm}$. A dashed line showing the results of linear interpolation is also shown. Lines in c are kernel-interpolated spatial profiles, created with a Gaussian kernel at different kernel widths. $\mathbf{d}$ is a close-up of the STN entry transition, showing the results of various methods of standard interpolation (linear, cubic spline, and cubic) compared to Gaussian kernel interpolation. Pearson correlation coefficients $(\boldsymbol{r})$ are shown in the figure legends where appropriate.

ficient analysis revealed that $\mathbf{D}=0.1 \mathrm{~mm}$ resulted in the best agreement (mean $r=0.891$ ) (fig. 3a). We suggest that $\mathbf{D}=0.1 \mathrm{~mm}$ is optimal because SI methods average adjacent points, resulting in smoother data that more closely matches additional smoothing gained from a largerthan-expected value of D. $r$ values decreased at either side of $\mathbf{D}=0.1 \mathrm{~mm}$; shorter and longer values suffered from under- and over-smoothing, respectively (data not shown). Consequently, we used $\mathbf{D}=0.1 \mathrm{~mm}$ to calculate all true spatial profiles for subsequent comparison.

\section{Determining the Optimal $\lambda$}

SI methods average only adjacent points with equal weighting. Because KI utilizes depth information directly 


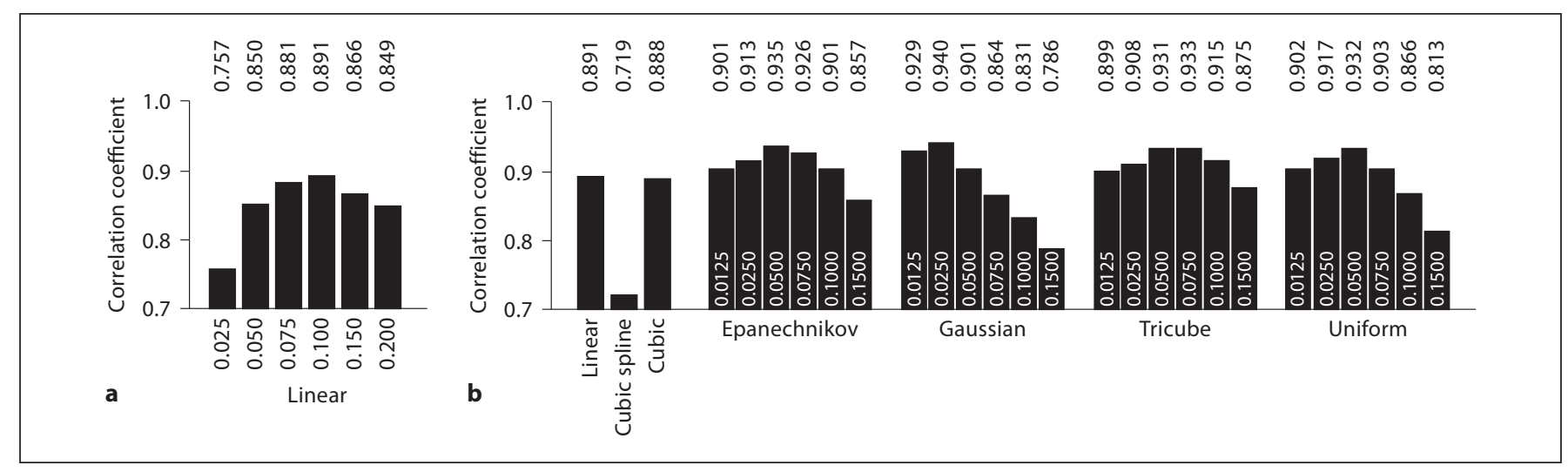

Fig. 3. Optimal depth window (D) and kernel width $(\lambda)$. a To compare between interpolated spatial profiles (created from temporal trends with $50 \%$ overlapping, 4 -second time windows) and true spatial profiles (produced from 50\% overlapping depth windows of length $\mathbf{D}$ ), we first had to determine the appropriate value for D. The optimal match occurred for $\mathbf{D}=0.01 \mathrm{~mm}$ (corresponding to an average $r=0.891$ for our dataset), with drop-offs on either side due to under- and over-smoothing. $\mathbf{b}$ The mean Pearson correlation coefficient is shown for various methods of interpolation, at the optimal value of $\mathbf{D}=0.01 \mathrm{~mm}$. SI methods (linear, cubic spline, and cubic) that interpolate between adjacent points only are the first three bars on the left. The remaining kernel-based interpolation methods are grouped by kernel function and plotted according to kernel width $\lambda$ (shown in white text within each bar). Regardless of kernel function, KI consistently produced superior results at values around $\lambda=0.05 \mathrm{~mm}$ and nearby values. The Gaussian kernel, due to its nonzero representation outside of the kernel width, was associated with superior values at lower $\lambda$. in its calculation, we hypothesized that KI would provide superior results and sought to show this quantitatively. However, the choice of the kernel width $\lambda$ changes the ultimate appearance of the KI spatial profile, with larger values resulting in a greater degree of smoothing (fig. 2c). Values of $\lambda$ less than $\mathbf{D}$ are most appropriate because each point that is incorporated into the kernel local average is itself derived from various depths. We estimated that $\lambda$ values of approximately $0.5 \mathrm{D}$ would be near optimal.

\section{Goodness of Fit: KI versus SI}

Figure $3 \mathrm{~b}$ shows correlation coefficients ( $\mathrm{r}$ values) for both $\mathrm{KI}$ and SI methods, after fixing $\mathbf{D}=0.1 \mathrm{~mm}$ and varying $\lambda$. Among the SI methods tested, linear interpolation performed the best by a slim margin over cubic interpolation, with mean $r$ values of 0.891 versus 0.888 , respectively. The poorest values were seen with cubic spline interpolation $(\mathrm{r}=0.719)$.

Linear interpolation can be viewed as the closest approximation to directly plotting feature output from time windows according to their depth stamps. However, linear interpolation resulted in a lower mean $r$ value than KI at optimal values of $\lambda$. Mean values of $r$ were $0.935,0.931$, and 0.932 for Epanechnikov, Tricube, and Uniform kernels, respectively, at $\lambda=0.05$ (half of the value of $\mathbf{D}$ ). For the Gaussian kernel, $r=0.940$ at $\lambda=0.025$. Neighboring values of $\lambda$ around these optimal values also produced $r$ values that were superior to linear interpolation. These results confirm our hypothesis that KI better approximates true spatial profiles under appropriate values of $\lambda$ that are relatively wide-ranging.

Comparison of correlation coefficients on a feature-byfeature basis revealed similar findings (fig. 4). The advantages of KI over SI, at the appropriate values of $\lambda(0.05 \mathrm{~mm}$ for Epanechnikov, Tricube, and Uniform kernels and $0.025 \mathrm{~mm}$ for the Gaussian kernel in this experiment) are a consistent finding, regardless of computational feature.

\section{Visualization}

Figure 5 shows examples of KI spatial profiles and the corresponding temporal trends from which they were generated. Localization is enhanced because the location and extent of the activity from STN and surrounding subcortical structures is standardized, obviating the need for a separate depth-time plot. Standardization also facilitates structural and functional comparison between different microelectrode tracks.

\section{Discussion}

Standard clinical practice in MER data acquisition results in irregularly sampled neurophysiological data with respect to depth. Consequently, feature activity trends are 


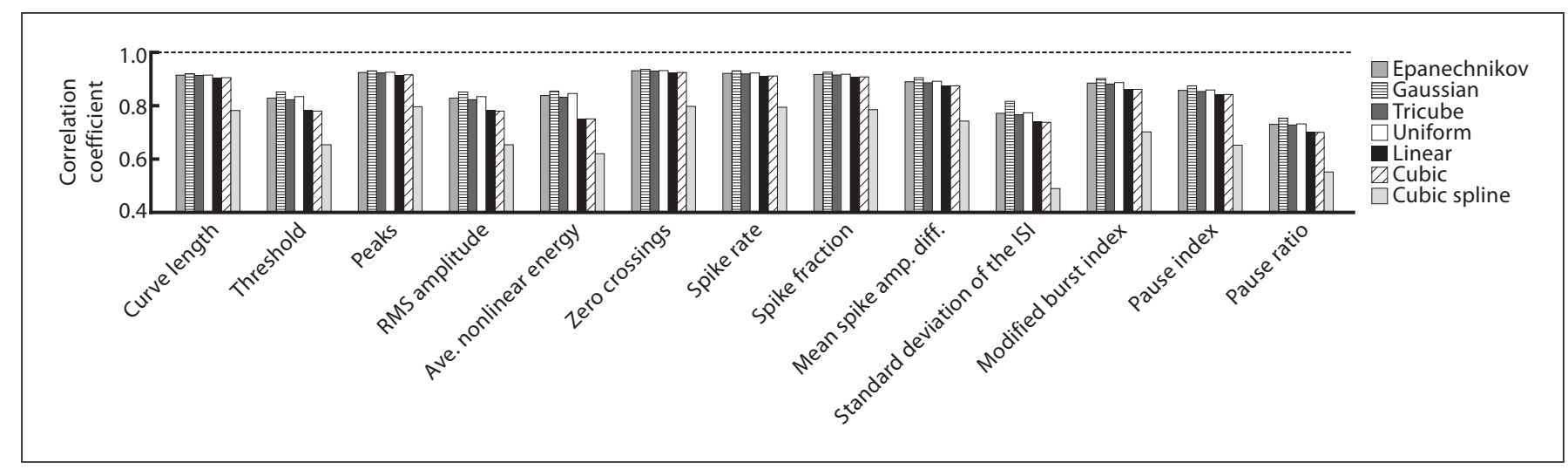

Fig. 4. Feature-wise comparison of interpolation methods. Comparison of interpolation methods at optimal values of $\mathbf{D}=0.01 \mathrm{~mm}$ and kernel width $\lambda=0.5 \mathrm{~mm}(0.025 \mathrm{~mm}$ for the Gaussian kernel) reveals slightly superior Pearson correlation coefficients for KI (Epanechnikov, Gaussian, Tricube, and Uniform) versus SI (linear, cubic, and cubic spline) across all features tested.

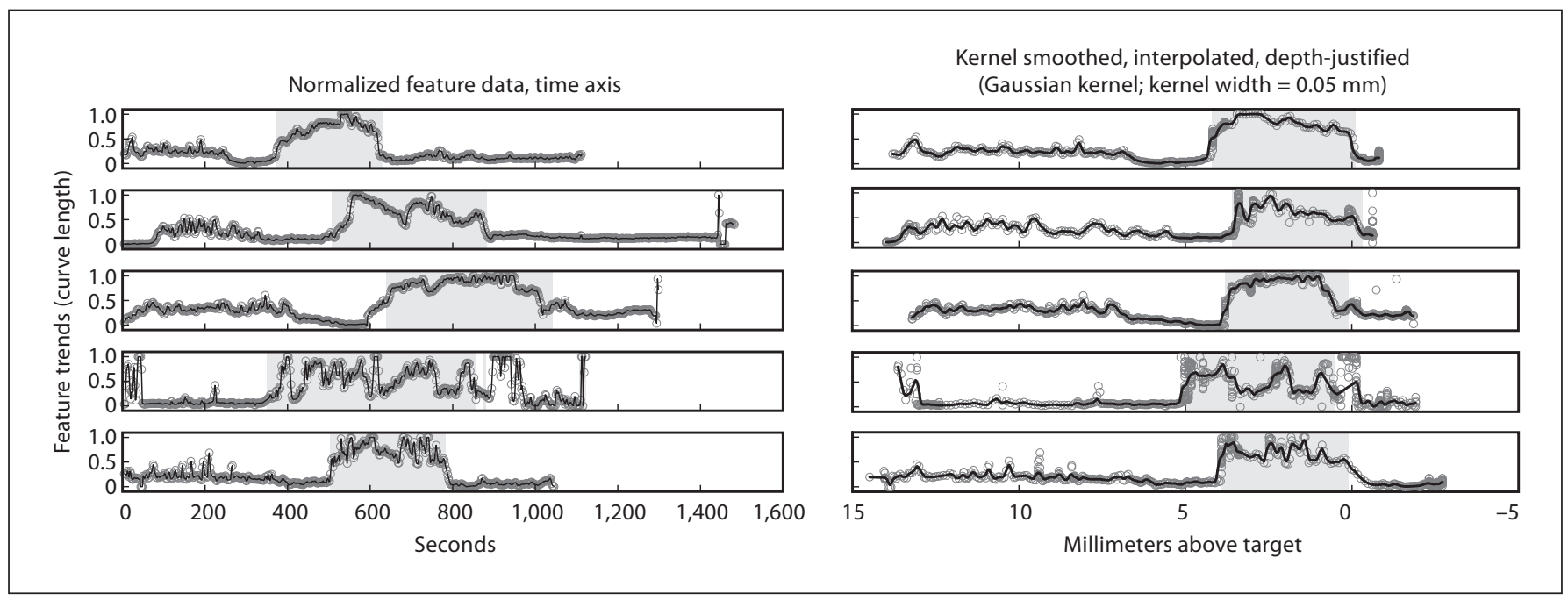

Fig. 5. KI examples. The top panels show feature activity from one particular feature calculation (curve length). Open circles represent normalized feature activity. STN boundaries are marked by gray boxes. Left panels show the original temporal trends. Right panels show the KI spatial profiles with a Gaussian kernel $(\lambda=0.05 \mathrm{~mm})$. The location and extent of STN and other subcortical structures on the KI profiles is standardized compared to the temporal trends, enhancing localization.

spatially nonlinear and distorted, making the task of functional localization utilizing these profiles more difficult. To correct this issue, we evaluated different interpolation methods to convert temporal trends to spatial profiles. Spatial profiles eliminate the need to correlate activity with a separate time-versus-depth plot. KI is an especially elegant, 'spatially aware' interpolation method that more readily handles irregularly sampled data and allows coordinate transformation in a statistically sound manner.

Though one can use depth windows to generate true spatial profiles, there are several disadvantages. First, depth windows are more cumbersome to implement, requiring safeguards to guarantee a minimum of data for a robust estimate of the spiking rate. Calculations within a depth interval must be updated if microelectrodes re- 
visit the location. Second, to change the resolution, one must change the width of the depth window, which requires recalculation of features along the entire microelectrode track. Third, physiological SN entry is an important clue for the neurophysiologist that the microelectrode tip is ventral to the STN. If one uses an automated approach with a temporal trend, this can be confirmed without having to penetrate too deeply into the $\mathrm{SN}$; a spatial profile would require deeper penetration to resolve a functional-anatomic boundary. Finally, perhaps the most important consideration is that a temporal trend is desirable for its ability to resolve changes in feature activity while the electrode is stationary, e.g. during sensorimotor testing to identify the STN. For these reasons, a solution such as interpolation that allows one to quickly convert from a temporal to a spatial representation is superior.

Our results show good outcomes with nearly all methods of interpolation. Regardless, KI is especially ideal because of the following advantages:

(1) KI spatial profiles were consistently superior in approximating true spatial profiles, independent of the feature used, though this superiority was marginal on correlation coefficient analysis. This superiority is likely directly related to the incorporation of depth during its calculation.

(2) KI performs spatial de-noising. This results in a more visually pleasing profile since transient temporal recording artifacts are averaged away when multiple features are extracted nearby. Spatially unaware methods are unable to do this.

(3) In some situations it may be desirable to know an interpolated feature value at a particular depth, which is easily achieved by KI but not by SI.

Both KI and SI methods are computationally efficient; in our experience, the time required to interpolate among hundreds to thousands of feature values on a circa-2009 laptop computer was negligible, allowing the operator to create interpolated spatial profiles rapidly in real-time. For KI, varying the kernel function and kernel width according to preference for a typical microelectrode track was near instantaneous, allowing rapid visualization of spatial profiles at different resolutions. It is important to note that simply plotting time window feature outputs versus their depth stamps (after averaging feature activity at common depths) is a degenerate case of KI with infinitesimal kernel widths.

One disadvantage of KI compared to SI is that an extra parameter $\lambda$ must be set. We attempted to pre-define it here by exploring different values of $\lambda$ among the differ- ent kernel functions. We found that the optimal value was approximately equal to the average distance travelled during each time window, and suggest using this value a priori when performing KI. Reassuringly, a relatively wide range of values for $\lambda$ around this optimum still produced superior correlation coefficients. A relatively narrower $\lambda$ is required when using the Gaussian kernel since (unlike the other kernel functions studied) the Gaussian function is represented beyond the kernel width.

These results and conclusions reflect our center's experience with our unique personnel and recording setup. The mathematics are universal and similar results should be seen at other institutions. However, the exact nonlinearity of microelectrode movement certainly varies among practitioners. This would result in variations in the magnitude of the differences between the interpolation methods seen here, as one would expect better correlation using KI with greater nonlinearity of movement. It is important to note that we did not compare our interpolation methods with traditional spatial profiles obtained by pausing the microelectrode for careful recording at intervals (i.e. zero-width depth windows), though good correlation is likely. Our goal was to evaluate interpolation of temporal trends (with proven localization value) to a depth axis, which in turn facilitates localization.

It is important to acknowledge that unavoidable spatial bias exists with irregularly sampled microelectrode data and therefore also any method using local spatial weighting. Whether one generates spatial profiles using depth windows, or KI with noninfinitesimal values of $\lambda$, a larger number of measurements at one location will skew the surrounding neighborhood's feature output towards the values from the over-represented location. Another unavoidable limitation is that variable spatial 'blurring' of neurophysiological data is unavoidable with irregular electrode velocities; the faster the microelectrode moves, the more uncertain the location from which neural activity originates is. These effects may be accounted for in future studies by utilizing dynamic kernel widths and shapes that weight adjacent physiology according to instantaneous electrode velocity.

\section{Conclusions}

Interpolation is an efficient and accurate method to transition between temporal and spatial representations of mobile microelectrode activity. Among the choices available, kernel methods offer a statistically sound 
method of creating interpolated spatial profiles that most closely match true spatial profiles created with depth windows. Automated localization is enhanced and standardized by the ability to rapidly switch between temporal and spatial representations of microelectrode activity that emphasize the functional and structural aspects of MER, respectively. This framework does not interfere with the traditional clinical task of MER localization, allowing clinicians to move the microelectrode as they see fit. These findings represent an incremental advance towards a real-time automated STN detection algorithm and 1-D structural/functional imaging with MER.

\section{Acknowledgements}

Stephen Wong was funded via the following grants: NIH CTSA grant No. KL2 RR 024132, NIH T32 DA022605-01, and a University of Pennsylvania Comprehensive Neuroscience Center collaborative grant. Shabbar Danish was funded through NIH grant No. T32 NS043126-03 and the Department of Veteran's Affairs Parkinson's Disease Research, Education, and Clinical Center (PADRECC).

\section{Disclosure Statement}

The algorithmic details of the feature profiles/activity maps presented here have been patented (US serial No. 12/441,973) through the Center for Technology Transfer at the University of Pennsylvania.

\section{References}

1 Gotman J: Automatic detection of seizures and spikes. J Clin Neurophysiol 1999;16: 130-140.

2 Wilson SB, Scheuer ML, Emerson RG, Gabor AJ: Seizure detection: evaluation of the Reveal algorithm. Clin Neurophysiol 2004;115: 2280-2291.

3 Benazzouz A, Breit S, Koudsie A, Pollak P, Krack P, Benabid AL: Intraoperative microrecordings of the subthalamic nucleus in Parkinson's disease. Mov Disord 2002; 17(suppl 3):S145-S149.

4 Israel Z, Burchiel K: Microelectrode Recording in Movement Disorder Surgery. New York, Thieme, 2004

5 Moran A, Bar-Gad I, Bergman H, Israel Z: Real-time refinement of subthalamic nucleus targeting using Bayesian decision-making on the root mean square measure. Mov Disord 2006;21:1425-1431.

-6 Falkenberg JH, McNames J, Burchiel KJ: Automatic microelectrode recording analysis and visualization of the globus pallidus interna and stereotactic trajectory. Stereotact Funct Neurosurg 2006;84:28-34.
7 Falkenberg JH, McNames J, Favre J, Burchiel $\mathrm{KJ}$ : Automatic analysis and visualization of microelectrode recording trajectories to the subthalamic nucleus: preliminary results. Stereotact Funct Neurosurg 2006;84:35-44, discussion 44-55.

8 Hutchison WD, Allan RJ, Opitz H, Levy R, Dostrovsky JO, Lang AE, Lozano AM: Neurophysiological identification of the subthalamic nucleus in surgery for Parkinson's disease. Ann Neurol 1998;44:622-628.

-9 Magnin M, Morel A, Jeanmonod D: Singleunit analysis of the pallidum, thalamus and subthalamic nucleus in parkinsonian patients. Neuroscience 2000;96:549-564.

10 Novak P, Daniluk S, Ellias SA, Nazzaro JM: Detection of the subthalamic nucleus in $\mathrm{mi}$ croelectrographic recordings in Parkinson disease using the high-frequency $(>500 \mathrm{~Hz})$ neuronal background: technical note. J Neurosurg 2007; 106:175-179.

- 11 Pesenti A, Rohr M, Egidi M, Rampini P, Tamma F, Locatelli M, Caputo E, Chiesa V, Bianchi A, Barbieri S, et al: The subthalamic nucleus in Parkinson's disease: power spectral density analysis of neural intraoperative signals. Neurol Sci 2004;24:367-374.

-12 Danish SF, Baltuch GH, Jaggi JL, Wong S: Determination of subthalamic nucleus location by quantitative analysis of despiked background neural activity from microelectrode recordings obtained during deep brain stimulation surgery. J Clin Neurophysiol 2008;25:98-103.
13 Favre J, Taha JM, Baumann T, Burchiel KJ: Computer analysis of the tonic, phasic, and kinesthetic activity of pallidal discharges in Parkinson patients. Surg Neurol 1999;51: 665-672, discussion 672-673.

14 Wong S, Baltuch GH, Jaggi JL, Danish SF: Functional localization and visualization of the subthalamic nucleus from microelectrode recordings acquired during DBS surgery with unsupervised machine learning. J Neural Eng 2009;6:026006.

15 Moyer JT, Danish SF, Keating JG, Finkel LH, Baltuch GH, Jaggi JL: Implementation of dual simultaneous microelectrode recording systems during deep brain stimulation surgery for Parkinson's disease: technical note. Neurosurgery 2007;60:ONSE177-178, discussion ONSE178.

-16 Danish SF, Jaggi JL, Moyer JT, Finkel L, Baltuch GH: Conventional MRI is inadequate to delineate the relationship between the red nucleus and subthalamic nucleus in Parkinson's disease. Stereotact Funct Neurosurg 2006;84:12-18.

17 Hastie T, Tibshirani R, Friedman J: The Elements of Statistical Learning: Data Mining, Inference, and Prediction. New York, Springer, 2001, pp 165-192. 